\title{
Comportamento sedentário em adolescentes atendidos pela Estratégia de Saúde da Família em Cuiabá, Brasil
}

\author{
Sedentary behaviour in adolescentes attended by the Family Health \\ Strategy in Cuiaba, Brazil
}

Marcelo Gomes Alexandre', Ageo Mário Cândido da Silva², Christianne de Faria Coelho-Ravagnani³

\begin{abstract}
Resumo
Analisamos a associação entre fatores relacionados ao domínio pessoal, ao ambiente físico e ao ambiente social com o comportamento sedentário (CS), pautando-se em pressupostos socioecológicos. Inicialmente cada variável independente foi testada separadamente com o desfecho - análises bivariadas através do teste Qui-quadrado de Pearson calculando-se a Razão de Prevalência bruta (RPb) e Intervalos de Confiança (IC) de $95 \%$. No processo das análises multivariáveis, utilizou-se a técnica de Regressão de Poisson múltipla e os pressupostos da utilização dos modelos hierarquizados. A análise final constituiu-se de 399 adolescentes (59\% meninas e $62 \% \geq 15$ anos) atendidos pela Estratégia de Saúde da Família de Cuiabá-MT. A prevalência de comportamento sedentário foi de 55\% para a amostra total. Os fatores: morar com o pai ( $\mathrm{RP}=1,08$; IC95\% $1,00 ; 1,17)$, escolaridade materna $<8$ anos $(\mathrm{RP}=0,89$; IC95\% $0,83 ; 0,96)$, consumo de álcool por familiares ( $\mathrm{RP}=1,14 ; \mathrm{IC} 95 \%$ $1,06-1,23)$, não conhecer locais para prática de atividade física (AF) $(\mathrm{RP}=1,09$; IC95\% 1,01;1,17) e não conhecer programas de saúde para jovens ( $\mathrm{RP}=1,10$; IC95\% 1,02;1,19) foram associados significativamente ao CS. Dos cinco fatores associados ao $\mathrm{CS}$, um fator relacionou-se ao ambiente físico (conhecer locais para prática de $\mathrm{AF}$ ) e quatro ao ambiente social (escolaridade materna, morar com o pai, consumo de álcool por familiares e conhecer programas de saúde para jovens). Os achados deste estudo sugerem a necessidade de intervenções para a redução do CS por parte da Estratégia de Saúde da Família, direcionadas não apenas ao contexto individual dos adolescentes, mas à família e ao ambiente físico dessa comunidade.
\end{abstract}

\section{Palavras-chave}

Comportamento do adolescente; Atenção Primária à Saúde; Promoção da saúde.

\begin{abstract}
The association between factors related to the personal domain, physical environment and social environment with sedentary behavior (SB) were analyzed, based on the socio-ecological premise. Initially each independent variable was tested separately with the outcome - bivariate analyzes - using the Pearson chi-square test, calculating the gross prevalence ratio (PSb) and confidence intervals (CI) of $95 \%$. In the process of multivariate analyzes, the multiple Poisson regression technique was used and assumptions for the use of hierarchical models. The analysis included 399 adolescents (59\% girls and 62\% $\geq 15 y$ ) attending the Family Health Strategy from Cuiaba-MT. Overall, the prevalence of sedentary behavior was 55\%. The factors: living with father $(P R=1.08 ; 95 \%$ $C I 1.00,1.17)$, maternal education $<8$ years $(P R=0.89 ; 95 \% C I$ $0.83,0.96)$, alcohol use by family members ( $P R=1.14$; $95 \% C I$ 1.06 to 1.23), lack of knowledge about locations of physical activity $(P A)(P R=1.09 ; 95 \%$ CI 1.01, 1.17) and lack of knowledge about health programs for young people ( $P R=1.10$; 95\% CI 1.02, 1.19) were significantly associated with $S B$. Among the five factors associated with $S B$, one factor was related to the physical environment (lack of knowledge about locations of physical activity) and four factors were related to the social environment (maternal education, living with father, alcohol use by family members and knowledge of health programs for young people). The findings of this study suggest the need for interventions to reduce $S B$ through Family Health Strategies, focused not only on the individual context of adolescents but also on the family members and the physical environment of the community.
\end{abstract}

\section{Keywords}

Adolescent Behavior; Primary Health Care; Health Promotion.
1 Instituto Federal de Mato Grosso, Sorriso, Mato Grosso, Brasil.

2 Universidade Federal de Mato Grosso, Programa de Pós-Graduação em Saúde Coletiva, Cuiabá, Mato Grosso, Brasil.

3 Universidade Federal de Mato Grosso, Programa de Pós-Graduação em Educação Física, Cuiabá, Mato Grosso, Brasil.

\section{Introdução}

A alta vulnerabilidade social dos adolescentes atendidos pela Atenção Primária à Saúde (APS) brasileira, decor- 
rente do baixo nível socioeconômico e escolaridade, menor acesso à informação e aos serviços médicos e à baixa participação desse segmento nas ações da Estratégia Saúde da Família (ESF) ${ }^{1}$, entre outros fatores os predispõem aos comportamentos de risco à saúde e consequentemente ao maior risco de Doenças Crônicas Não Transmissíveis (DCNT), caracterizando-os como um público específico e que merece investigação.

Nesse sentido, o comportamento sedentário (CS), um construto que apresenta correlatos e determinantes próprios ${ }^{2}$, associando-se com vários danos à saúde, tais como níveis elevados de peso corporal, doenças metabólicas, baixa auto-estima, dificuldades em relacionamentos sociais, consumo de tabaco e álcool ${ }^{3}$, configura-se como mais uma questão de saúde pública, visto que, tem apresentado prevalências elevadas tanto no Brasil, por exemplo em Cuiabá $58,1 \%{ }^{4}$ como em outros países ${ }^{3}$.

Sabendo que estilos de vida saudáveis, se adquiridos na adolescência tendem a permanecer na idade adulta ${ }^{5}$, torna-se viável conhecer os fatores que determinam esse comportamento e implementar intervenções com vistas a não somente combater os prejuízos à saúde nessa etapa da vida mas também fomentar comportamentos saudáveis em idades mais avançadas.

A literatura aponta para uma relação entre fatores sociais, ambientais e a saúde das comunidades ${ }^{6}$. Contudo, grande parte das ações em saúde pública ainda focam em aspectos individuais, limitando as chances de sucessos dessas intervenções. Desse modo, a análise dos determinantes dos comportamentos em saúde, incluindo o CS, sob o enfoque socioecológico permite o melhor planejamento das intervenções, pois consideram as interações dinâmicas entre pessoas, ambientes físicos e sociais ${ }^{6,7}$.

O olhar socioecológico desses determinantes, particularmente na APS e conseguintemente na ESF, sua maior vertente nacional, é de extrema relevância, pois estes modelos de atenção priorizam ações de promoção da saúde e prevenção de doenças voltadas principalmente ao contexto familiar e comunitário, valorizando o território (espaço físico), o espaço social e relacional dos indivíduos.

Contudo, até onde se tem conhecimento, não há no país levantamentos sobre os fatores determinantes do CS em adolescentes atendidos pela ESF. Vale destacar que estudos envolvendo a área CS na APS brasileira são extremamente escassos. Os estudos nacionais que investigaram esses fatores não distinguem usuários de sistemas públicos de saúde $e^{4,8}$.

Diante do exposto, o presente estudo analisou a prevalência e os fatores associados ao CS de adolescentes vinculados à ESF, pautando-se em premissas socioecológicas?.

\section{Métodos}

O presente estudo foi conduzido como parte do projeto de pesquisa intitulado "A saúde do adolescente na Atenção Primária de Cuiabá - Mato Grosso: sob a ótica da multidisciplinaridade", proposto pelo Programa de Educação pelo Trabalho para Saúde/PET-SAÚDE9. Trata-se de um estudo transversal conduzido em 2011. A população alvo estimada em 7.014 sujeitos (identificados a partir de registros - Fichas A - oriundos das Unidades Básicas de Saúde/UBS), composta por adolescentes (de 12 a 19 anos de ambos os sexos) ${ }^{10}$ residentes no município de Cuiabá e cadastrados em Estratégias de Saúde da Família (ESF) que possuíam a atuação do PET-Saúde. A cidade possui uma área territorial de $3.291,812 \mathrm{~km}^{2}$ sendo geograficamente dividida em quatro regiões, o PET-Saúde estava implantado em 19 UBS abrangendo todas as regionais (Regional Sul, Norte, Leste e Oeste), no ano de 2011 sua população urbana era de 540.814 habitantes $^{11}$.

A seleção da amostra foi elaborada em múltiplos estágios: partindo da listagem de todos os adolescentes inseridos nas 19 ESF, recorreu-se à amostragem estratifi- 
cada para a divisão dos indivíduos em partes de forma que a representatividade de cada unidade na população permanecesse constante; os demais procedimentos basearam-se nos preceitos da amostragem sistemática, sorteando um ponto de partida e estabelecendo um determinado intervalo, respectivamente, para cada ESF.

Preconizou-se para o cálculo do tamanho da amostra: nível de confiança de 95\%; erro amostral tolerável de cinco pontos percentuais; prevalência estimada em 50\%. Obteve-se um tamanho amostral de 364 adolescentes, sobre os quais foi acrescentado o percentual de $20 \%$, equivalente a um efeito de delineamento amostral de 1,2, diante de possíveis perdas e recusas, definindo-se uma amostra de 437 adolescentes para o estudo. Devido a perdas e recusas de 38 sujeitos, a amostra final foi composta por 399 adolescentes, mantendo-se acima do padrão mínimo estabelecido / proporção esperada.

A concordância formal para participação no estudo se deu através da obtenção da assinatura do adolescente ou de seu responsável (para aqueles com idade abaixo de 18 anos). Todos os procedimentos de intervenção foram aprovados pelo Comitê de Ética do Hospital Universitário Júlio Müller - protocolo 693/2009.

Os participantes foram investigados mediante a utilização de um questionário adaptado ${ }^{12}$ do "Global School-Based Student Health Survey" (que para o presente estudo conteve acréscimos de questões referentes a relação entre a ESF e os adolescentes - Figura 1) desenvolvido pela Organização Mundial de Saúde (OMS) em colaboração com outros organismos internacionais ${ }^{13}$. As aplicações foram conduzidas nos respectivos domicílios de forma individual e sem a aproximação dos pais, por aplicadores previamente capacitados e prontamente dispostos ao esclarecimento de possíveis dúvidas. Os adolescentes preencheram o instrumento sem identificação pessoal, porém codificados. Após obtenção dos dados, estes foram duplamente digitados em planilha do aplicativo Excel, e a consistência interna testada no pacote estatístico SPSS versão 20.0 for Windows (SPSS Inc., Chicago, Estados Unidos).

A variável dependente do estudo foi a exposição ao CS, que se referiu ao tempo gasto sentado (assistindo televisão, jogando no computador, conversando com amigos, jogando cartas ou dominó), em um dia normal, acima de duas horas, > 2h/ $\mathrm{dia}^{3}$. As variáveis independentes (figura 2) - fatores referentes ao domínio pessoal (características biogenéticas, psicológicas e comportamentais), ambiente físico e ambiente social - foram escolhidas e agrupadas levando em consideração as questões do "Global School-Based Student Health Survey" que se enquadravam nas perspectivas do modelo socioecológico de promoção da saúde 7 . Inicialmente cada variável independente foi testada separadamente com o desfecho - análises bivariadas através do teste Qui-quadrado de Pearson calculando-se a Razão de Prevalência bruta (RPb) e Intervalos de Confiança (IC) de 95\%.

No processo das análises multivariáveis, utilizou-se a técnica de Regressão de Poisson múltipla e os pressupostos da utilização dos modelos hierarquizados ${ }^{14}$. As variáveis, hierarquizadas em blocos (figura 3), foram inseridas manualmente na modelagem. Os blocos foram testados de modo distal-proximal em relação a variável dependente - ou seja, primeiro foi inserido o bloco I e assim sucessivamente, entendendo que os blocos mais distantes da variável dependente são os que diretamente tem um menor efeito no sentido de causar um problema de saúde ou influenciar em um comportamento de saúde ${ }^{14}$ - e o efeito de cada variável sobre o desfecho foi interpretado como ajustado para as variáveis pertencentes aos blocos hierarquicamente anteriores e para os efeitos das variáveis que se encontravam no mesmo bloco. Na primeira fase ocorreu a modelagem dentro de cada bloco, somente as variáveis que demonstraram um valor de $\mathrm{p}<0,10$ foram consideradas 
FIGURA 1 - Questões que foram utilizadas para identificar a relação entre adolescentes e a Estratégia de Saúde da Família, no município de Cuiabá, estado de Mato Grosso. Brasil, 2011.

\begin{tabular}{|c|c|}
\hline Questões & Alternativas \\
\hline Você já ouviu falar do Programa de Saúde da Família (PSF)? & $\begin{array}{l}\text { Sim } \\
\text { Não }\end{array}$ \\
\hline $\begin{array}{l}\text { Com que frequência você vai à Unidade de Saúde (posto de } \\
\text { saúde)? }\end{array}$ & $\begin{array}{l}\text { Eu nunca fui ao posto de saúde } \\
\text { Raramente } \\
\text { As vezes } \\
\text { Sempre }\end{array}$ \\
\hline Qual é o principal motivo que você vai ao posto de saúde? & $\begin{array}{l}\text { Eu nunca fui ao posto de saúde } \\
\text { Quando estou doente } \\
\text { Para fazer exames preventivos } \\
\text { Por que sou convidado pela equipe de saúde } \\
\text { Para pegar remédio } \\
\text { Para pegar camisinhas } \\
\text { Para tomar vacina } \\
\text { Outros }\end{array}$ \\
\hline $\begin{array}{l}\text { Alguma vez alguém do posto de saúde (médico, enfermeiro, } \\
\text { agente de saúde) fez visita na sua casa para falar sobre a SUA } \\
\text { saúde? }\end{array}$ & $\begin{array}{l}\text { Sim } \\
\text { Não } \\
\text { Não lembro }\end{array}$ \\
\hline $\begin{array}{l}\text { No seu bairro você conhece algum programa de saúde para } \\
\text { jovens e adolescentes? }\end{array}$ & $\begin{array}{l}\text { Sim } \\
\text { Não } \\
\text { Não lembro }\end{array}$ \\
\hline $\begin{array}{l}\text { Você já foi convidado a participar de algum programa de saúde } \\
\text { para os jovens e adolescentes? }\end{array}$ & $\begin{array}{l}\text { Sim } \\
\text { Não } \\
\text { Não lembro }\end{array}$ \\
\hline $\begin{array}{l}\text { Você participa de algum programa de saúde para os jovens e } \\
\text { adolescentes? }\end{array}$ & $\begin{array}{l}\text { Sim } \\
\text { Não } \\
\text { Não lembro }\end{array}$ \\
\hline $\begin{array}{l}\text { Alguma vez a sua escola desenvolveu alguma atividade em } \\
\text { conjunto com o posto de saúde do seu bairro? }\end{array}$ & $\begin{array}{l}\text { Sim } \\
\text { Não } \\
\text { Não lembro }\end{array}$ \\
\hline $\begin{array}{l}\text { Se o posto de saúde te convidasse a participar de alguma } \\
\text { palestra ou atividade/grupo para adolescentes você aceitaria? }\end{array}$ & $\begin{array}{l}\text { Sim } \\
\text { Não } \\
\text { Não sei }\end{array}$ \\
\hline $\begin{array}{l}\text { De onde você obtém informações sobre a sua saúde (marque a } \\
\text { principal fonte)? }\end{array}$ & $\begin{array}{l}\text { De sites da internet } \\
\text { Das propagandas da TV } \\
\text { Do posto de saúde } \\
\text { Dos meus pais ou parentes } \\
\text { Dos meus amigos } \\
\text { Da escola } \\
\text { De outras fontes de informação } \\
\text { Dos meus pais ou parentes } \\
\text { Dos meus amigos } \\
\text { Da escola } \\
\text { De outras fontes de informação }\end{array}$ \\
\hline
\end{tabular}

TV: televisão.

nas fases seguintes. A modelagem da segunda fase consistiu na união dos blocos I e II. Na terceira fase ocorreu a união dos blocos I, II e III, onde foram considerados significativamente associados ao CS somente os fatores com valor de $\mathrm{p}<0,05$. Todas as análises foram realizadas utilizando-se o pacote estatístico STATA versão 10.0 (Stata Corp., College Station, Estados Unidos).

\section{Resultados}

No presente estudo foram excluídos os adolescentes não encontrados após três visitas em dias alternados $(n=18)$ e sem autorização dos pais ou responsáveis $(n=20)$. Desse modo, a amostra final constituiu-se de 399 adolescentes, sendo que, 59\% eram do sexo feminino e aproximadamente $62 \%$ estavam compreendidos na faixa etária de 15 a 19 anos. Aproximadamente 55\% deles apresentaram o CS (tabela 1). 
FIGURA 2 - Definição das variáveis e categorias adotadas para análise dos dados.

\begin{tabular}{|c|c|c|}
\hline Variável & Categoria & Medida utilizada \\
\hline \multicolumn{3}{|l|}{ Domínio pessoal } \\
\hline Sexo & $\begin{array}{l}\text { Masculino } \\
\text { Feminino }\end{array}$ & Auto-resposta \\
\hline Idade & $\begin{array}{l}<15 \text { anos } \\
\geq 15 \text { anos }\end{array}$ & $\begin{array}{l}\text { Classificação com base nos critérios propostos pela World } \\
\text { Health Organization (1986) }\end{array}$ \\
\hline Escolaridade & $\begin{array}{l}\geq 8 \text { anos } \\
<8 \text { anos }\end{array}$ & $\begin{array}{l}\text { Completou o ensino fundamental ou ingressou no ensino } \\
\text { médio/superior; estar inserido no ensino fundamental ou ter } \\
\text { deixado os estudos antes de completar o ensino fundamental }\end{array}$ \\
\hline Etnia/cor & $\begin{array}{l}\text { Branco } \\
\text { Não branco }\end{array}$ & $\begin{array}{l}\text { Relatar qual etnia/cor considera ser: branco, preto, pardo, } \\
\text { mulato, moreno, indígena, amarelo, outro. }\end{array}$ \\
\hline Consumo de álcool & $\begin{array}{l}\text { Não } \\
\text { Sim }\end{array}$ & $\begin{array}{l}\text { Ter feito uso/consumo pelo menos uma vez nos últimos trinta } \\
\text { dias }\end{array}$ \\
\hline Consumo de frutas & $\begin{array}{l}\text { Sim } \\
\text { Não }\end{array}$ & $\begin{array}{l}\text { Consumiu suco natural ou fruta uma vez por semana, nos } \\
\text { últimos trinta dias }\end{array}$ \\
\hline Consumo de verduras & $\begin{array}{l}\text { Sim } \\
\text { Não }\end{array}$ & $\begin{array}{l}\text { Consumiu verduras uma vez por semana, nos últimos trinta } \\
\text { dias }\end{array}$ \\
\hline Consumo de refrigerante & $\begin{array}{l}\text { Sim } \\
\text { Não }\end{array}$ & $\begin{array}{l}\text { Consumiu refrigerante uma vez por semana, nos últimos } \\
\text { trinta dias }\end{array}$ \\
\hline Qualidade do sono & $\begin{array}{l}\geq \text { Boa } \\
<\text { Boa }\end{array}$ & $\begin{array}{l}\text { Auto-avaliação da qualidade do sono; opções: ruim, regular, } \\
\text { boa, muito boa e excelente }\end{array}$ \\
\hline Gostar de AF & $\begin{array}{l}\text { Concorda } \\
\text { Discorda }\end{array}$ & Afirmação quanto a gostar de fazer atividades físicas \\
\hline $\begin{array}{l}\text { Considerar AF importante } \\
\text { para saúde }\end{array}$ & $\begin{array}{l}\text { Sim } \\
\text { Não }\end{array}$ & Auto-resposta \\
\hline $\begin{array}{l}\text { Participação em aulas de } \\
\text { Educação Física }\end{array}$ & $\begin{array}{l}\text { Sim } \\
\text { Não }\end{array}$ & $\begin{array}{l}\text { Durante uma semana normal participa de aulas de Educação } \\
\text { Física. }\end{array}$ \\
\hline Sexualmente ativo & $\begin{array}{l}\text { Não } \\
\text { Sim }\end{array}$ & Relaciona-se sexualmente \\
\hline Consumo de tabaco & $\begin{array}{l}\text { Não } \\
\text { Sim }\end{array}$ & Ter feito uso pelo menos uma vez nos últimos trinta dias \\
\hline $\begin{array}{l}\text { Estágios de Mudança de } \\
\text { Comportamento }\end{array}$ & $\begin{array}{l}\text { Ativo } \\
\text { Inativo }\end{array}$ & $\begin{array}{l}\text { Se considera nos estágios de ação e manutenção; ou nos } \\
\text { estágios pré-contemplativos, contemplativos e preparação. }\end{array}$ \\
\hline Percepção de saúde & $\begin{array}{l}\geq \text { Boa } \\
<\text { Boa }\end{array}$ & $\begin{array}{l}\text { Auto-avaliação da saúde; opções: muito ruim, ruim, boa, } \\
\text { excelente. }\end{array}$ \\
\hline $\begin{array}{l}\text { Frequenta Unidades Básicas } \\
\text { de Saúde }\end{array}$ & $\begin{array}{l}\text { Sim } \\
\text { Não }\end{array}$ & Se frequenta uma unidade de saúde. \\
\hline \multicolumn{3}{|l|}{ Ambiente social } \\
\hline Mora com o pai & $\begin{array}{l}\text { Sim } \\
\text { Não }\end{array}$ & Mora com o pai. \\
\hline Mora com a mãe & $\begin{array}{l}\text { Sim } \\
\text { Não }\end{array}$ & Morar com a mãe. \\
\hline Escolaridade materna & $\begin{array}{l}\geq 8 \text { anos } \\
<8 \text { anos }\end{array}$ & $\begin{array}{l}\text { Completou o ensino fundamental ou ingressou no ensino } \\
\text { médio/superior; estar inserida no ensino fundamental ou ter } \\
\text { deixado os estudos antes de completar o ensino fundamenta. }\end{array}$ \\
\hline Recebe auxilio financeiro & $\begin{array}{l}\text { Sim } \\
\text { Não }\end{array}$ & $\begin{array}{l}\text { Receber algum auxilio financeiro, podendo ser federal, } \\
\text { estadual ou municipal. }\end{array}$ \\
\hline $\begin{array}{l}\text { Conhece programas } \\
\text { de saúde para jovens e } \\
\text { adolescentes }\end{array}$ & $\begin{array}{l}\text { Sim } \\
\text { Não }\end{array}$ & Auto-reposta. \\
\hline Familiares ativos & $\begin{array}{l}\text { Sim } \\
\text { Não }\end{array}$ & $\begin{array}{l}\text { Ter entre os moradores da residência alguém fisicamente } \\
\text { ativo. }\end{array}$ \\
\hline $\begin{array}{l}\text { Consumo de álcool por } \\
\text { familiares }\end{array}$ & $\begin{array}{l}\text { Não } \\
\text { Sim }\end{array}$ & Pais ou responsáveis tomam bebidas alcoólicas. \\
\hline $\begin{array}{l}\text { Consumo de álcool por } \\
\text { amigos }\end{array}$ & $\begin{array}{l}\text { Não } \\
\text { Sim }\end{array}$ & Possuir amigos que tomam bebidas alcoólicas. \\
\hline
\end{tabular}

Continua... 
... continua.

\begin{tabular}{lll}
\hline Variável & Categoria & Medida utilizada \\
\hline Prática religiosa & Sim & Considera-se praticante da religião a que pertence. \\
& Não & \\
\hline Trabalha & $\operatorname{Sim}$ & Exerce função remunerada. \\
& Não & \\
\hline Estuda & $\operatorname{Sim}$ & Auto-reposta. \\
\hline Não & & \\
\hline $\begin{array}{l}\text { Possui horta/árvore frutífera } \\
\text { residencial }\end{array}$ & Sim & Existência de horta/árvore frutífera na residência \\
\hline $\begin{array}{l}\text { Conhece local para prática } \\
\text { de atividade física }\end{array}$ & Sim & Não bairro onde o sujeito mora existe ou não alguma \\
& Não & $\begin{array}{l}\text { academia de ginástica/musculação, clube, escola de esporte, } \\
\text { praças, campos, quadras para a prática de atividade física. }\end{array}$ \\
\hline
\end{tabular}

FIGURA 3 - Estrutura conceitual desenvolvida para apoiar a análise hierárquica relativa ao comportamento sedentário.

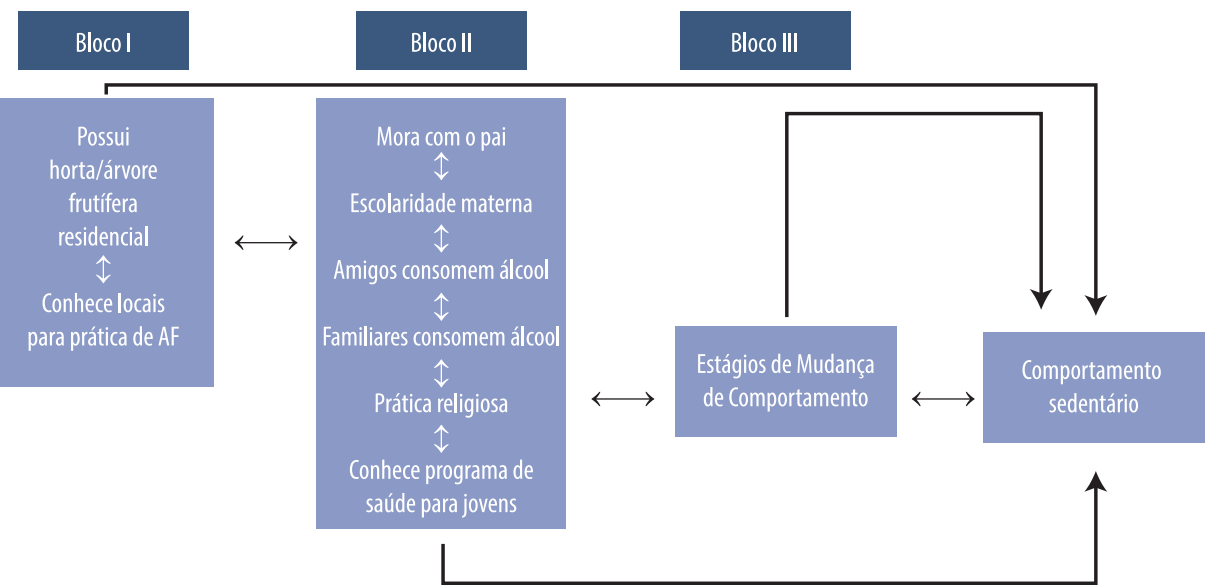

A partir dos modelos de regressão hierárquica relacionado ao CS (tabela 2), nota-se que quando as variáveis estavam ajustadas entre si em seus respectivos blocos - primeiro modelo - os fatores não conhecer locais para prática de $\mathrm{AF}$, escolaridade materna $<8$ anos, ter familiares que consomem álcool e não conhecer programas de saúde para jovens foram associados significativamente com o comportamento sedentário em adolescentes. Havendo o ajuste entre os blocos - modelos II e III - a variável não morar com o pai tornou-se significativamente associada ao comportamento sedentário.

TABELA 1 - Características de adolescentes atendidos pela Estratégia Saúde da Família, no município de Cuiabá, estado de Mato Grosso. Brasil, 2011.

\begin{tabular}{lllcc}
\hline \multicolumn{1}{c}{ Variável } & & $\mathrm{n}$ & $\%$ & IC 95\% \\
\hline Trabalho remunerado & Não & 368 & 92,70 & $89,3-94,8$ \\
\hline & Sim & 29 & 7,30 & $5,23-10,7$ \\
\hline Recebe auxílio do governo & Sim & 117 & 29,77 & $25,0-34,2$ \\
\hline \multirow{2}{*}{ Sexo } & Não & 276 & 70,23 & $65,8-75,0$ \\
\hline & Masculino & 163 & 40,85 & $36,1-46,0$ \\
\hline Idade & Feminino & 236 & 59,15 & $54,0-63,9$ \\
\hline & 12 a 14 & 152 & 38,29 & $33,4-43,2$ \\
\hline
\end{tabular}


... continua.

\begin{tabular}{lllll}
\hline \multicolumn{1}{c}{ Variável } & & $\mathrm{n}$ & $\%$ & IC 95\% \\
\hline Escolaridade materna & $\geq 8$ anos & 174 & 44,62 & $38,8-48,7$ \\
\hline Cor/etnia & $<8$ anos & 216 & 55,38 & $51,2-61,2$ \\
\hline & Branco & 55 & 13,96 & $10,7-17,7$ \\
\hline Comportamento Sedentário & Outros & 339 & 86,04 & $82,3-89,3$ \\
\hline & $>$ 2horas/dia & 217 & 54,94 & $49,5-59,5$ \\
\hline
\end{tabular}

n: Número de participantes; \%: Percentual de participantes; IC95\%: Intervalo de Confiança de 95\%.

TABELA 2 - Regressão com modelos hierarquizados para identificação de fatores associados ao comportamento sedentário de adolescentes atendidos pela Estratégia Saúde da Família, no município de Cuiabá, estado de Mato Grosso. Brasil, 2011.

\begin{tabular}{|c|c|c|c|}
\hline \multirow[t]{2}{*}{ Blocos de variáveis independentes } & $\begin{array}{c}\text { Modelo I (blocos 1, } 2 \text { e } \\
3 \text { isolados) }\end{array}$ & Modelo II (blocos 1+2) & $\begin{array}{c}\text { Modelo III (blocos } \\
1+2+3)\end{array}$ \\
\hline & RPal (IC95\%) & RPall (IC95\%) & RPall (IC95\%) \\
\hline \multicolumn{4}{|l|}{ Bloco 1} \\
\hline \multicolumn{4}{|c|}{ Possui horta/árvore frutifera residencial } \\
\hline Sim & 1,00 & & \\
\hline Não & $1,04(0,96-1,12)$ & & \\
\hline \multicolumn{4}{|l|}{ Conhece locais para prática de AF } \\
\hline Sim & 1,00 & 1,00 & 1,00 \\
\hline Não & $1,10(1,01-1,19)^{* *}$ & $1,09(1,01-1,17)^{* *}$ & $1,09(1,01-1,17)^{* *}$ \\
\hline \multicolumn{4}{|l|}{ Bloco 2} \\
\hline \multicolumn{4}{|l|}{ Mora com o pai } \\
\hline Sim & 1,00 & 1,00 & 1,00 \\
\hline Não & $1,07(0,99-1,16)^{*}$ & $1,08(1,00-1,17)^{* *}$ & $1,08(1,01-1,17)^{* *}$ \\
\hline \multicolumn{4}{|l|}{ Escolaridade materna } \\
\hline$\geq 8$ anos & 1,00 & 1,00 & 1,00 \\
\hline$<8$ anos & $0,88(0,82-0,95)^{* *}$ & $0,89(0,83-0,96)^{* *}$ & $0,89(0,83-0,96)^{* *}$ \\
\hline \multicolumn{4}{|l|}{ Consumo de álcool por amigos } \\
\hline Sim & 1,00 & & \\
\hline Não & $1,02(0,94-1,11)$ & & \\
\hline \multicolumn{4}{|l|}{ Consumo de álcool por familiares } \\
\hline Sim & 1,00 & 1,00 & 1,00 \\
\hline Não & $1,15(1,06-1,25)^{* *}$ & $1,14(1,06-1,23)^{* *}$ & $1,14(1,06-1,23)^{* *}$ \\
\hline \multicolumn{4}{|l|}{ Prática religiosa } \\
\hline Sim & 1,00 & & \\
\hline Não & $1,05(0,96-1,14)$ & & \\
\hline \multicolumn{4}{|c|}{ Conhece programas de saúde para jovens } \\
\hline Sim & 1,00 & 1,00 & 1,00 \\
\hline Não & $1,13(1,04-1,23)^{* *}$ & $1,10(1,02-1,19)^{* *}$ & $1,10(1,02-1,19)^{* *}$ \\
\hline \multicolumn{4}{|l|}{ Bloco 3} \\
\hline \multicolumn{4}{|l|}{ EMC } \\
\hline Ativo & 1,00 & & \\
\hline Inativo & $1,08(0,95-1,24)$ & & \\
\hline
\end{tabular}

RPal: Razão de Prevalência ajustada entre variáveis do mesmo bloco; RPall: Razão de Prevalência ajustada entre variáveis do mesmo bloco e entre blocos; AF: Atividade física; EMC: Estágios de Mudança de Comportamento; os valores assinalados com * representam associações com $\mathrm{p}$-valor <0,10; aqueles com ** apresentam associações significativas $(p<0,05)$. 


\section{Discussão}

Em termos gerais, nossos resultados apontam para a prevalência acima de $50 \%$ de CS entre os adolescentes vinculados à ESF, todavia, ficando abaixo do resultado encontrado anteriormente na mesma cidade $(58,1 \%)$ e da média nacional $(78 \%)$ 4,8 . Além disso, denotam o caráter multifatorial do comportamento, uma vez que, no modelo final de regressão notamos a associação significativa com cinco fatores dispostos em diferentes níveis do modelo socioecológico da promoção da saúde 7 . Um fator relacionou-se ao ambiente físico (conhecer locais para prática de AF) e quatro ao ambiente social (escolaridade materna, consumo de álcool por familiares, morar com o pai e conhecer programas de saúde para jovens).

Em um estudo realizado também na região Centro-Oeste ${ }^{4}$, apenas dois fatores considerados como do ambiente social estiveram associados ao CS, enquanto que, houve quatro relativos ao domínio pessoal e um ao ambiente físico. Frequentemente estudos que observam o CS dão maior atenção a fatores pertencentes ao domínio pessoal ${ }^{4,15,16}$. O fato é que a verificação de fatores do ambiente físico e social se demonstra como mais complexa e ainda não há uma devida padronização de instrumentos para suas respectivas verificações, dificultando assim tal tarefa.

Além disso, o agrupamento dos correlatos em diferentes categorias é muitas vezes distinto entre as pesquisas, reduzindo a possibilidade de comparação com o presente estudo. Por exemplo, num estudo de revisão ${ }^{8}$, foram feitos cinco grupos para analisar os correlatos do comportamento sedentário, sendo que, pelo menos três deles poderiam estar inseridos no que nós denominamos como domínio pessoal. Talvez este fato explique a grande diversidade de determinantes e correlatos do CS e a notável diferença nos resultados dos estudos.

A literatura aponta associações com sexo ${ }^{17}$, idade ${ }^{4,18,19}$, alimentação ${ }^{20}$, consumo de álcool ${ }^{4,21}$ e tabaco ${ }^{21}$, autoeficácia ${ }^{22,23}$, percepção de saúde ${ }^{21}$, dentre outros fatores, todos considerados como individuais/pessoais. Entre os fatores relativos ao ambiente social aparecem com destaque, a escolaridade dos pais ${ }^{4,18,19,22}$, incentivo materno para ser ativo ${ }^{24}$ e regras dos pais sobre assistir $\mathrm{TV}^{22,23}$.

Embora os estudos demonstrem que adolescentes de classe mais elevada, bem como os de escolas privadas, apresentam-se mais propensos à comportamentos sedentários ${ }^{4,25}$, no presente estudo, a prevalência de CS (55\%; IC95\% 49,5;59,5) foi semelhante a um estudo realizado na região Centro Oeste ${ }^{4}$ que continha adolescentes de diferentes classes sociais, sugerindo que a facilidade de acesso aos meios tecnológicos como TV, computadores, celulares e vídeo-games já esteja presente na vida dos adolescentes vinculados à ESF. Outro achado importante foi o fato de que adolescentes que não conheciam programas de saúde para jovens apresentavam maior prevalência deste comportamento. Até onde se tem conhecimento, não há estudos nesta perspectiva no Brasil ${ }^{8}$.

No contexto familiar, os fatores consumir álcool, não morar com o pai e escolaridade materna > 8 anos, estiveram associados ao CS dos adolescentes. A literatura aponta que o fato de não morar com os familiares não resulta em associação com $\mathrm{CS}^{26}$, por outro lado, regras paternas demonstraram associação significativa ${ }^{23}$. Nesse sentido, pode-se supor que um adolescente que não mora com o pai não possui do mesmo modo regras paternas e portanto é mais suscetível a apresentar o CS. Outrossim, em relação a família, em países desenvolvidos, a escolaridade materna foi inversamente associada ao $\mathrm{CS}^{18,22}$, enquanto que neste estudo adolescentes que possuíam mães com escolaridade > 8 anos, apresentaram maior prevalência de CS, pressupondo que pais com mais elevada escolaridade têm maior poder de compra e, com isso, maior facilidade para adquirir aparelhos eletrônicos ${ }^{4,19}$. 
Faz-se necessário apontar que, a variável morar com o pai passou a ser associada significativamente ao CS após ajuste entre blocos. Esse resultado confirma um dos pressupostos do modelo socioecológico, a existência de uma interação entre variáveis de diferentes níveis de influência. Contudo, os resultados do presente estudo devem ser analisados tendo em vista algumas limitações. O estudo apresenta um delineamento transversal, o que não permite inferir causalidade; e, o modelo socioecológico utilizado para o CS não é específico para explicar tal comportamento, todavia é adequado do ponto de vista conceitual, pois é relativo a comportamentos relacionados à promoção de saúde.

Ainda, as variáveis inseridas no presente estudo não são consideradas como pertencentes às principais teorias aplicadas aos estudos do comportamento, contudo, nas perspectivas do modelo socioecológico fornecem um quadro da amplitude de como esses comportamentos devem ser enfrentados. Diferenças entre nossos resultados e os observados na literatura podem ser em razão das análises estatísticas utilizadas e do modelo conceitual adotado.

Apesar das reconhecidas limitações, o presente estudo, ao nosso conhecimento é o primeiro a analisar o CS levantando importantes considerações para a intervenção na ESF ${ }^{8}$.

Assim, nossos resultados sugerem que a melhor adequação e a maior aproximação dos serviços da APS com os adolescentes se faz imprescindível, pois embora haja necessidade do atendimento a estes, muitas vezes a ESF não os tem alcançado ${ }^{1}$. Somando-se a isso, a expansão ou efetivação/atuação do Programa Saúde da Escola poderá contribuir para o estabelecimento de tais premissas, visto que a escola é o ambiente ideal para promoção de saúde ${ }^{27}$.

$\mathrm{Na}$ ótica da APS, caberia a ESF atuar no sentido de informar os familiares e adolescentes sobre os prejuízos do CS, pressupondo que a baixa escolaridade materna e a falta da presença do pai incidem nesse comportamento pelo caminho da falta de orientação.

Embora a falta de prática de atividade física e o CS se apresentarem na literatura como construtos diferentes ${ }^{28}$, o fator referente ao conhecimento de locais para a prática de AF esteve associado ao CS. Particularmente em Cuiabá, os locais públicos para a prática de $\mathrm{AF}$ apresentam diversas inadequações no ambiente construído, ambiente estético, além de falta de segurança e poluição; quanto aos locais considerados particulares, estes exigem taxas de mensalidades elevadas dificultando assim a utilização por parte dos adolescentes ${ }^{29}$. A inter-relação entre o ambiente e a AF é intuitiva ${ }^{30}$, ou seja, se houver espaços (para tal prática) os adolescentes os utilizarão, evitando também uma longa permanência em atividades sedentárias em suas residências. Contudo, não podemos apontar como intervenção a adequação, revitalização ou criação de novos espaços para as práticas de AF nas áreas de abrangência das ESF. Estudos longitudinais devem ser elaborados permitindo assim inferirmos causalidade.

A prevalência de comportamento sedentário foi de $55 \%$. Os fatores: morar com o pai ( $\mathrm{RP}=1,08$; IC95\% 1,00;1,17), escolaridade materna < 8 anos ( $\mathrm{RP}=0,89$; IC95\% $0,83 ; 0,96)$, consumo de álcool por familiares ( $\mathrm{RP}=1,14$; IC95\% 1,06-1,23), não conhecer locais para prática de atividade física (AF) (RP=1,09; IC95\% 1,01;1,17) e não conhecer programas de saúde para jovens ( $\mathrm{RP}=1,10$; IC95\% 1,02;1,19) foram associados significativamente ao CS. Dos cinco fatores associados ao CS, um fator relacionou-se ao ambiente físico (conhecer locais para prática de $\mathrm{AF}$ ) e quatro ao ambiente social (escolaridade materna, morar com o pai, consumo de álcool por familiares e conhecer programas de saúde para jovens). 


\section{Contribuição dos autores}

Contribuições dos autores: ALEXANDRE MG, SILVA AMC e Ravagnani CFC participaram da concepção e delineamento do estudo, análise e interpretação dos dados, redação, revisão e aprovação final do manuscrito, e são responsáveis por todos os aspectos do trabalho, incluindo a garantia de sua precisão e integridade.

\section{Referências}

1. Duarte SJH, Urel DR, Zorman IBS, Alexandre MG, Ravagnani CFC. A prática do autocuidado à saúde na perspectiva dos adolescentes. Rev Bras Enferm. 2014; 8(5):321-7.

2. Biddle SJH. Sedentary Behavior. Am J Prev Med. 2007;33(6): 502-4.

3. Salmon J, Tremblay MS, Marshall SJ, Hume C. Health Risks, Correlates, and Interventions to Reduce Sedentary Behavior in Young People. Am J Prev Med. 2011; 41(2):197-206.

4. Dias PJP, Domingos IP, Ferreira MG, Muraro AP, Sichieri R, Silva RMVG. Prevalência e fatores associados aos comportamentos sedentários em adolescentes. Rev Saúde Públ. 2014; 48( 2):266-74.

5. Telama R, Yang X, Leskinen E, Kankaanpa A, Hirvensalo M, Tammelin T, et al. Tracking of Physical Activity from Early Childhood through Youth into Adulthood. Med Sci Sports Exerc. 2014;46(5):955-62.

6. Linke SE, Robinson CJ, Pekmezi D. Applying Psychological Theories to Promote Healthy Lifestyles. Am J Lifestyle Med. 2014;8(4):4-14.

7. Stokols D. Establishing and maintaining healthy environments: toward a social ecology of health promotion. Am Psychol. 1992; 47(1):6-22.

8. Guerra PH, Farias Junior JC, Florindo AA. Sedentary behavior in Brazilian children and adolescentes: a systematic review. Rev Saúde Públ. 2016; 50(9): 1-15.

9. Silva VG, Ribeiro TC, Machado AD, Duarte SJH, Coelho-Ravagnani CF. A educação física no programa de educação pelo trabalho para a saúde de Cuiabá-MT. Rev Bras Ativ Fís Saúde. 2012; 17(2):121-4.

10. World Health Organization. Young people's health - a challenge for society. Geneva: World Health Organization; 1986 [citado $2011 \mathrm{Fev} 2$ ] . Disponível em: http://apps.who. int/iris/bitstream/10665/41720/1/WHO_TRS_731.pdf

11. Instituto Brasileiro de Geografia e Estatística. População urbana: Cuiabá (MT). Rio de Janeiro: Instituto Brasileiro de Geografia e Estatística; 2010. [citado 2016 Mar 1]. Disponível em:http://www.censo2010.ibge.gov.br/sinopse/webservice/frm_pu_hom_ mul.php?codigo $=510340$.

12. Legnani E, Legnani RFS, Morgenroth A. Reprodutibilidade e objetividade de um questionário sobre comportamentos de risco à saúde em adolescentes. Coleção Pesquisa em Educação Física. 2008; 7(3): 351-6.

13. Centers for Disease Control and Prevention. Global School-based Student Health Survey (GSHS). Atlanta: Centers for Disease Control and Prevention; 2015 [citado 2011 Fev 2]. Disponível em: url:http:// www.cdc.gov/GSHS/

14. Victora CG, Huttly SR, Fuchs SC, Olinto MTA. The Role of Conceptual Frameworks in Epidemiological Analysis: A Hierachical Approach. Int J Epidemiol. 1997; 26( 1):224-7.

15. Horst KVD, Paw MJC, Twisk JWR, Mechelen WV. A Brief Review on Correlates of Physical Activity and Sedentariness in Youth. Med Sci Sports Exerc. 2007; 39(80):1241-50.

16. Craggs C, Corder K, Sluijs EMFV, Griffin SJ. Determinants of change in physical activity in children and adolescents: a systematic review. Am J Prev Med. 2011; 40:645-58.

17. Leatherdale ST, Ahmed R. Screen-based sedentary behaviours among a nationally representative sample of youth: are Canadian kids couch potatoes? Chronic Dis Inj Can. 2011; 31(4):141-6.

18. Rey-López JP, Tomas C, Vicente-Rodriguez G, Gracia-Marco L, Gracia-Marco L, JiménezPavón D, et al. Sedentary behaviours and socio-economic status in Spanish adolescents: the AVENA study. Eur J Public Health. 2010; 21( 2):151-7.

19. Campagnolo PDB, Vitolo MR, Gama CM. Fatores Associados ao Habito de Assistir TV em Excesso Entre Adolescentes. Rev Bras Med Esporte.2008;14( 3):197-200.

20. Ramos E, Costa A, Araújo J, Severo M, Lopes C. Effect of television viewing on food and nutrient intake among adolescents. Nutrition.2013; 5(7):1362-7.

21. Kim JY. The Nonlinear Association Between Internet Using Time for Non-Educational Purposes and Adolescent Health. J Prev Med Public Health.2012;45 (1):37-46. 
22. Gebremariam MK, Totland TH, Andersen LF, Bergh IH, Bjelland M, Grydeland M, et al. Stability and change in screen-based sedentary behaviours and associated factors among Norwegian children in the transition between childhood and adolescence. BMC Public Health.2012;12(104):1-9.

23. Cillero IH, Jago R, Sebire S. Individual and social predictors of screen-viewing among Spanish school children. Eur J Pediatr. 2011;170:93-102.

24. Bauer KW, Nelson MC, Boutelle KN, Neumark-Sztainer D. Parental influences on adolescents physical activity and sedentary behavior: longitudinal findings from Project EAT. II. Int J Behav Nutr Phys Act.2008;5(12):1-7.

25. Oliveira TC, Silva AAM, Santos CJN, Silva JS, Conceição SIO. Atividade física e sedentarismo em escolares da rede pública e privada de ensino em São Luís. Rev Saúde Pública. 2010;44(6):996-1004.

26. Silva KS, Nahas MV, Peres KG, Lopes AS. Fatores associados à atividade física, comportamento sedentário e participação na Educação Física em estudantes do Ensino Médio em Santa Catarina, Brasil. Cad. Saúde Públ. 2009; 25(10):2187-200.

27. Ball K, Timperio AF, Crawford DA. Understanding environmental influences on nutrition and physical activity behaviors: where should we look and what should we count? Int J Behav Nutr Phys Act.2006 ;3(33):1-8.

28. Greca JPA, Silva DAS, Loch MR. Physical activity and sreen time in children and adolescents in a médium size tow in the South of Brazil. Rev Paul Pediatr. 2016; 1-7.

29. Silva IJO, Alexandre MG, Ravagnani FCP, Silva JVP, Coelho-Ravagnani CF. Atividade física: espaços e condições ambientais para sua prática em uma capital brasileira. R. Bras. Ci. e Mov. 2014; 22(3):53-62.

30. Hino AAF, Reis RS, Florindo AA. Ambiente construído e atividade física: uma breve revisão dos métodos de avaliação. Rev Bras Cineantropom Desempenho Hum. 2010; 12(5), 387-94.

ENDEREÇO PARA

CORRESPONDÊNCIA

MARCELO GOMES ALEXANDRE

marcelomesalex@gmail.com
Avenida dos Universitários, S/N

(Instituto Federal de Mato Grosso).

Bairro: Residencial Santa Clara.

CEP: 78890000;

Sorriso-MT, Brasil.

Telefone: (66) 99642-9256.
RECEBIDO 22/03/2016

REVISADO 18/07/2016

APROVADO $18 / 07 / 2016$ 